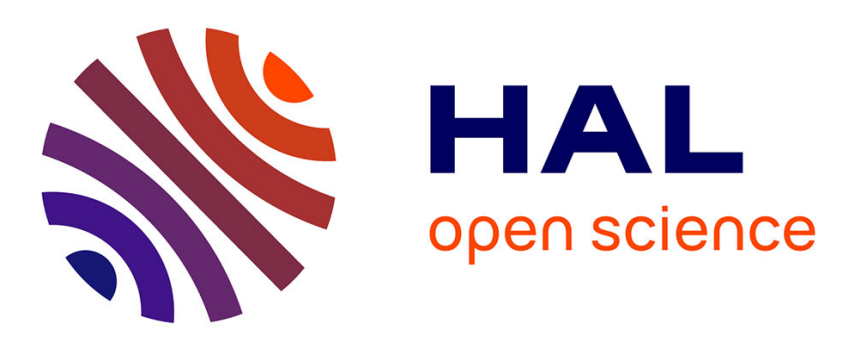

\title{
Monte Carlo simulation of primary recrystallization and annealing twinning
}

W Wang, A.L. Helbert, F Brisset, M H Mathon, T Baudin

\section{To cite this version:}

W Wang, A.L. Helbert, F Brisset, M H Mathon, T Baudin. Monte Carlo simulation of primary recrystallization and annealing twinning. Acta Materialia, 2014, 10.1016/j.actamat.2014.08.032 . hal03317762

\section{HAL Id: hal-03317762 \\ https://hal.science/hal-03317762}

Submitted on 7 Aug 2021

HAL is a multi-disciplinary open access archive for the deposit and dissemination of scientific research documents, whether they are published or not. The documents may come from teaching and research institutions in France or abroad, or from public or private research centers.
L'archive ouverte pluridisciplinaire HAL, est destinée au dépôt et à la diffusion de documents scientifiques de niveau recherche, publiés ou non, émanant des établissements d'enseignement et de recherche français ou étrangers, des laboratoires publics ou privés. 


\title{
Monte Carlo simulation of primary recrystallization and annealing twinning
}

W. Wang ${ }^{1}$, A. L. Helbert ${ }^{1}$, F. Brisset ${ }^{1}$, M.H. Mathon ${ }^{2}$, T. Baudin ${ }^{1}$

\author{
${ }^{1}$ Université Paris-Sud, ICMMO, CNRS UMR 8182, 91405 Orsay Cedex, France \\ ${ }^{2}$ Laboratoire Léon Brillouin, (CEA-CNRS), CEA Saclay, 91191 Gif sur Yvette, France
}

\begin{abstract}
.
Formation of annealing twins has been studied from the beginning of $20^{\text {th }}$ century. The related mechanisms as growth accident, nucleation of twins and nucleation of partial Shockley loops were suggested. The simulation was also realized by molecular dynamics simulations at the atomic scale. In this paper, a microscopic scale simulation of primary recrystallization and twinning by Monte Carlo approach was realized for a nickel alloy. Different twin morphologies were simulated. It showed a possibility of dependence of grain growth direction on twin formation during annealing. The incoherent $\Sigma 3$ and $\Sigma 9$ boundaries formation is verified as the indirect outcome after coherent $\Sigma 3$ formation.
\end{abstract}

\section{Keywords: Monte Carlo simulation, primary recrystallization, twinning, nickel alloy}

\section{Introduction}

The first observation of annealing twins can be traced back to the beginning of the $20^{\text {th }}$ century [1]. With the technology and material developments, it was largely observed during the annealing of the FCC materials with low stacking fault energy. The related mechanisms have also been studied for decades. Combining the proposed mechanisms [2-4], the annealing twin formation was defined as a nucleation of partial Shockley loops at the $\{111\}$ ledges (or emission of partial dislocations) from the migrating grain boundaries (GBs) [5-6], including the formation of fivefold twin in nanocrystalline materials [7]. This has been supported by molecular dynamics simulations [8-9].

From Gleiter's theory of [3], the twinning probability depends both on the annealing temperature and on the difference of Gibbs' free energy between the growing and the shrinking grains [3]. Pande et al. [10] found that the number of twins per grain depends on the grain size (not directly on temperature), and on the driving force. However, in these theories, the Gibbs' free 
energy gradient or the driving force was only related to the GB energy. They were then modified by Cahoon et al. [11] and Li et al. [12], who introduced the stored energy effect.

Moreover, with the Gleiter's model [3] the annealing twin was formed as an accident when the migrating GB was parallel to a $\{111\}$ plane of a growing grain. This theory was verified by a recent study based on transmission electron microscope observations (TEM) [13] and shown that the twin formation depends on the direction of grain growth. All of these studies show that the annealing twinning is an important mechanism related to primary recrystallization.

As a statistical and stochastic approach, Monte Carlo (MC) simulations are largely used for primary recrystallization and grain growth. However, the twin formation has not yet been introduced in this type of simulation, especially using the orientation image microscopy (OIM ${ }^{\mathrm{TM}}$ ) maps as input data, which contain the microstructure, the position of each pixel and their orientation.

Before the development of field emission gun-scanning electron microscopy (FEG-SEM), it was difficult to obtain an OIM map of the deformed state due to the resolution limit. Despite this problem, Caleyo et al. [14] have tried to simulate the primary recrystallization from an OIM map measured by SEM, but the non-defined pixels were attributed the orientations considering the global texture of the deformed material. Baudin et al. [15] have also run simulations using TEM data. Note that the input data could also come from finite element modelling of a deformed state [16]. After the development of FEG-SEM, the MC simulations directly from OIM map became feasible [17-18].

In the present paper, the authors will realize a microscopic simulation of the primary recrystallization through MC approach. The experimental data of a deformed material, obtained by electron backscatter diffraction (EBSD) system installed on a FEG-SEM will be used as input data. The multiple twinning will also be introduced with considering the variation of stored energy and the direction of grain growth.

\section{Material and initial structure}

A nickel alloy after hot forging and a homogenization process was employed [19]. After these treatments, this material presents a recrystallized and homogenous microstructure. The mean grain size is about $17 \mu \mathrm{m}$ without considering the twins. 
A sample cut from this material, was deformed by cold rolling with $60 \%$ reduction. Its deformed microstructure was analyzed by an EBSD system installed on a FEG-SEM. This analysis was carried out on the transverse plane (Rolling Direction RD - Normal Direction ND). The acquisition step was $200 \mathrm{~nm}$ based on a hexagonal grid. Then an EBSD map of $50 \times 50 \mu \mathrm{m}^{2}$ was chosen as the initial microstructure for simulation, whose texture is in good agreement with the global texture (Figure 1). The main texture components are Copper $\{112\}<111\rangle$ and the $a$ -fibre (from Goss $\{011\}<100>$ to Brass $\{011\}<211>$ ). This global texture was analyzed by neutron diffraction at the Laboratoire Léon Brillouin (CEA Saclay, France) on the diffractometer $6 \mathrm{~T} 1$.

The distribution of stored energy within this chosen microstructure was obtained, with the help of line broadening measurements of neutron diffraction peaks [20-21] and the calculation of kernel average misorientation (KAM) [22-23].

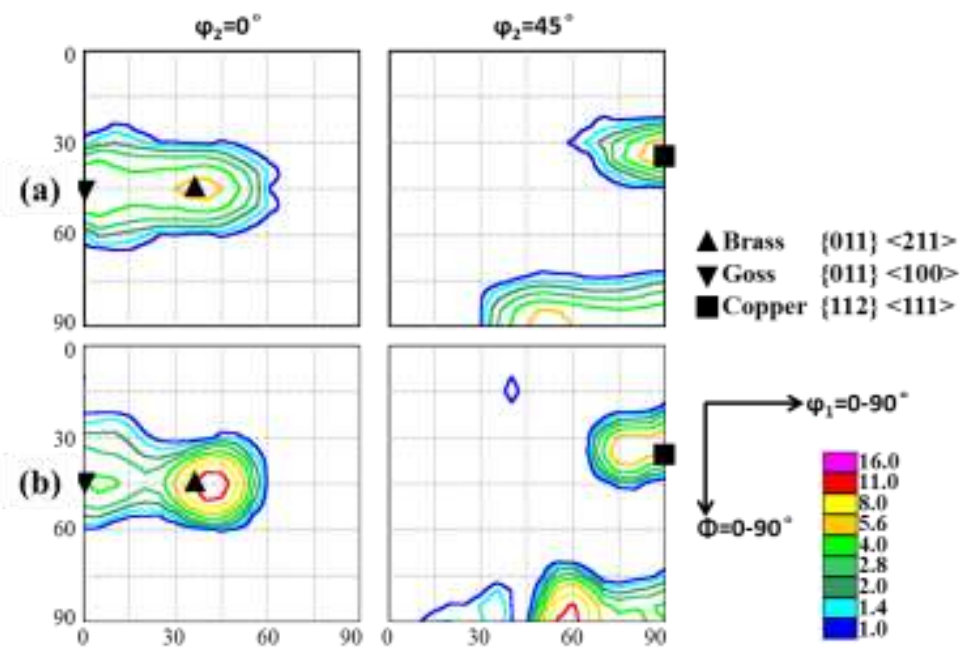

Figure 1: (a) Bulk texture (neutron diffraction) of the deformed sample after $60 \%$ cold rolling, and (b) local texture (OIM) of the initial microstructure for the simulation.

Firstly, using the neutron diffraction, the stored energy after deformation was determined as a function of grain orientation $H\left(\varphi_{1}, \phi, \varphi_{2}\right)$, and those of the three main components are shown in figure 2. With considering the measurement errors, the stored energy is almost identical for every component. Therefore, in this simulation, the stored energy was considered as a parameter independent of grain orientation. So instead of considering $H\left(\varphi_{1}, \phi, \varphi_{2}\right)$, a mean value $H_{\text {neutron }}$ 
was calculated with equation 1 .

$$
H_{\text {neutron }}=\sum_{i=1}^{n} F\left(\varphi_{1 i}, \phi_{i}, \varphi_{2 i}\right) \square H\left(\varphi_{1}, \phi, \varphi_{2}\right)
$$

$F\left(\varphi_{1 i}, \phi_{i}, \varphi_{2 i}\right)$ is the volume fraction of every orientation calculated with the LaboTex software from the orientation distribution function (ODF). The ODF is determined by using the discrete ADC method [24]. This mean value allows us to have a global vision of the stored energy in this deformed material.

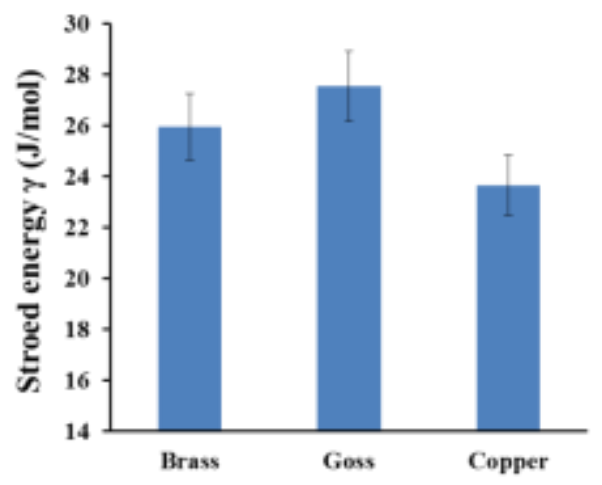

Figure 2: Stored energy of the main components after cold rolling with $60 \%$ reduction.

Moreover, the local deformation within the microstructure was calculated by the KAM parameter obtained from EBSD data. The kernel size is chosen as the $1^{\text {st }}$ neighboring pixels. The minimum misorientation is chosen as $15^{\circ}$ to define the GB. For every pixel, if the misorientation between it and any of its $1^{\text {st }}$ neighboring pixel is larger than this value, this neighboring pixel will be considered as belonging to another grain, and its misorientation will be removed from the KAM calculation. After this calculation, every pixel i has a KAM value $\theta_{i}$. According to that, the pixels can be color coded to obtain the KAM map, which will show the deformation distribution, associated to geometrical necessary dislocations, within the microstructure. As shown in figure 3, the local deformation is always around the high angle grain boundaries (HAGBs) (angle $>15^{\circ}$ ) (red lines) and the distribution is almost the same in every grain. 

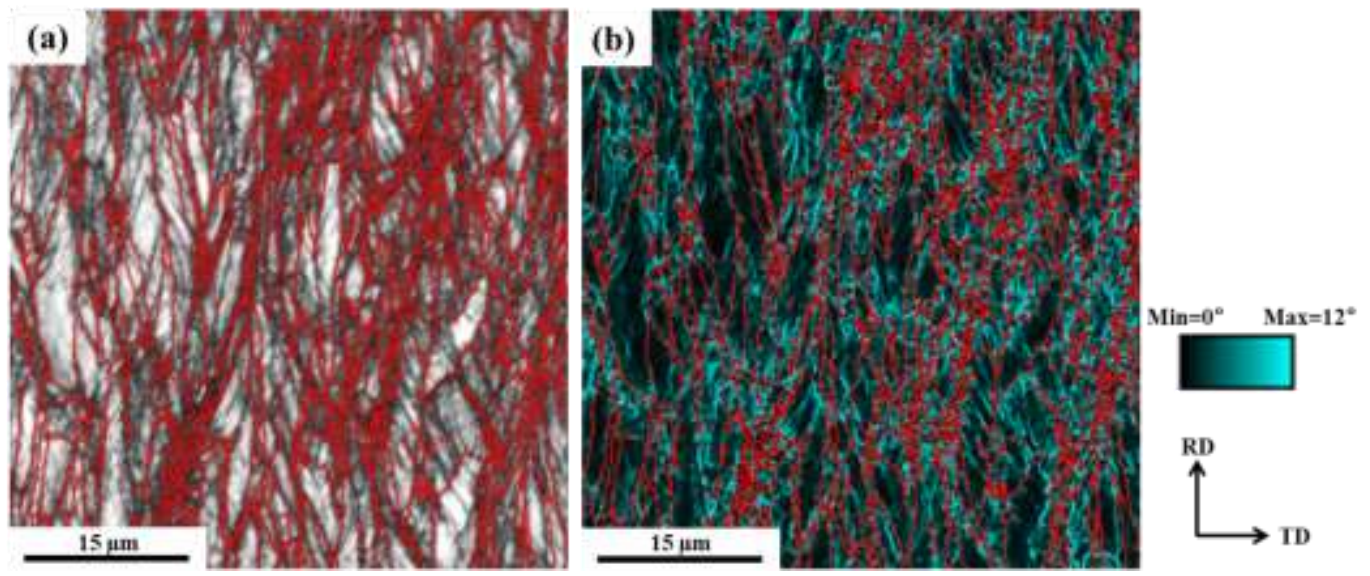

Figure 3: (a) EBSD map (Image Quality - IQ) of the initial microstructure and (b) KAM map of the same area. The red lines show the HAGBs.

From the KAM value $\theta_{i}$ of all the pixels, the maximum $\theta_{\max }$ and the minimum $\theta_{\min }$ misorientation within the microstructure can be detected. Then the stored energy of every pixel is distributed accordingly to the following rules:

The $H_{\text {neutron }}$ was defined as the maximum stored energy $\left(H_{\max }\right)$ in the deformed microstructure. The minimum stored energy $\left(H_{\min }\right)$ is chosen as $10 \%$ of $H_{\max }$. This minimum energy is not set to zero since zero is kept for the recrystallized pixels that will appear during the simulation of the recrystallization.

For every pixel $\mathrm{i}$, if its KAM value $\theta_{i}$ is equal to $\theta_{\max }$, then its stored energy $H_{i}$ is equal to $H_{\max }$;

If $\theta_{i}$ is equal to $\theta_{\min }$, then $H_{i}$ is equal to $H_{\min }$;

If $\theta_{i}$ has an intermediate value between $\theta_{\max }$ and $\theta_{\min }$, its $H_{i}$ is distributed by equation 2 .

$$
H_{i}=H_{\min }+\left(H_{\max }-H_{\min }\right) \frac{\theta_{i}-\theta_{\min }}{\theta_{\max }-\theta_{\min }}
$$

\section{MC simulation model}

In the MC simulations and for every calculation, a pixel i will be firstly chosen randomly. The simulation will then calculate the growth probabilities of its neighboring pixels towards itself 
(Figure 4a) [25-26]. The recrystallization corresponds to the growth of the neighbor pixel that has the highest probability (as the first neighbor in figure 4a).
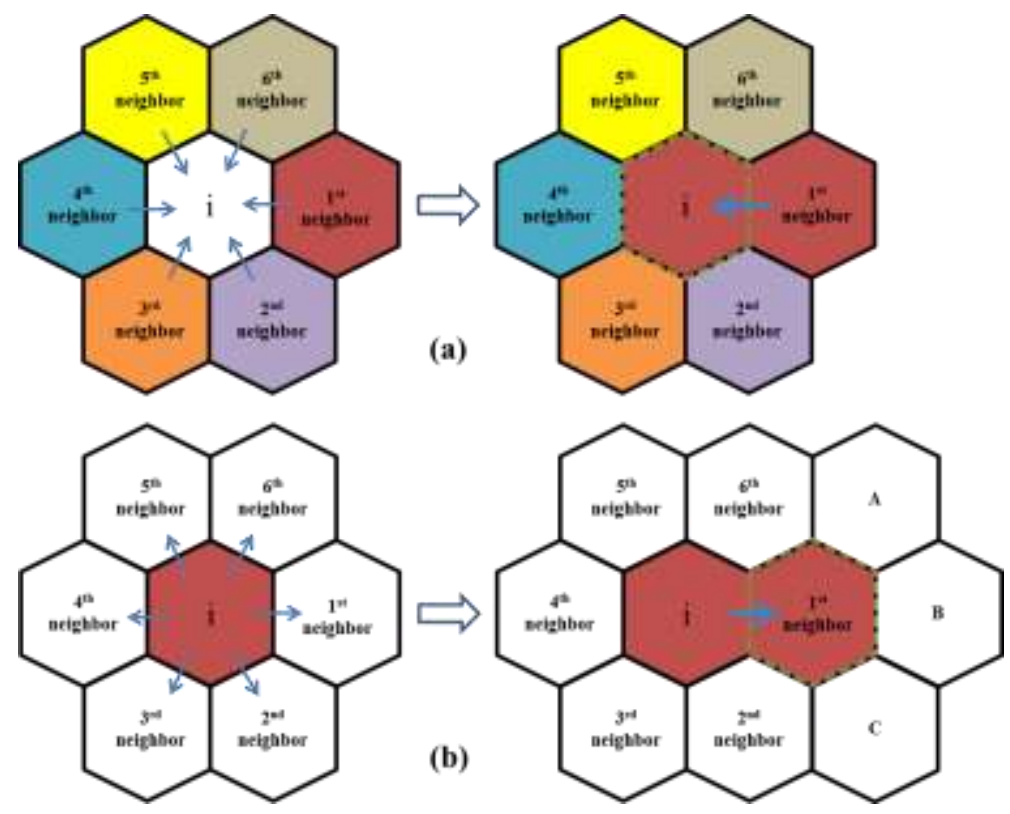

Figure 4: (a) Typical model of MC simulation: for a chosen pixel i, the recrystallization correspond to the growth of one neighboring pixel towards itself, which has the highest probability (as the growth of the $1^{\text {st }}$ neighbor towards the pixel i); (b) our model: for a chosen pixel $i$, the recrystallization correspond to its growth towards one neighboring pixel which has the highest probability (as the growth of pixel i towards the $1^{\text {st }}$ neighbor).

In our simulation, the twinning will be introduced during primary recrystallization. If the typical model is used, every neighbor has the twinning probability towards the pixel i. Moreover, every neighbor has four <111> axes as the possible twinning systems. So for every calculation, 24 twinning systems (4 axes x 6 neighbors) should be considered. In our model, the recrystallization corresponds to the growth of one chosen pixel i towards one of its neighbors with the highest possibility (as the first neighbor, figure $4 \mathrm{~b}$ ). Therefore, only the 4 axes of the chosen pixel i will be considered as the twinning systems.

In addition, the MC simulation was used only for the primary recrystallization and associated twinning during annealing at $900^{\circ} \mathrm{C}$. Indeed, the experimental results were shown in a previous paper [19], and the recovery is considered negligible during annealing. Therefore, the recovery is not introduced in the present study. More, the grain growth after complete primary recrystallization is not simulated here. 


\subsection{Primary recrystallization model}

The primary recrystallization is simulated in two parts: nucleation and growth of formed nuclei.

\subsubsection{Nucleation}

The recovery is an important process for the subsequent nucleation, as well as the time for nucleation and the distribution of nuclei. However, in our simulation, there is no recovery process. Instead of it, the critical number of nuclei $C N_{\text {nuclei }}$ is used as a condition of nucleation. As a new recrystallized grain directly provides from the growth of a nucleus, the number of nuclei can be estimated experimentally from the number of grains in the sample after complete recrystallization.

The critical nucleus size is about $200 \mathrm{~nm}$, observed experimentally by TEM [13]. As this value corresponds to the acquisition step size of an EBSD analysis, every pixel in the initial microstructure can be considered as a possible nucleus. To be a real nucleus, there are two critical conditions on this pixel:

- It depends on the probability of its growth towards one of its neighboring pixels (explained in the next section);

- The number of existing nuclei is still lower than the critical value $C N_{\text {nuclei }}$.

\subsubsection{Growth of the nuclei}

During the simulation, the growth of a nucleus corresponds to its growth towards the deformed area. The method described in [27] was used with some modifications. For a chosen pixel i (belonging to a nucleus), the driving force for its growth depends on the stored energy gradient $\Delta H_{i j}$, the GB energy $\gamma\left(\theta_{i j}\right)$ and the mobility $M\left(\theta_{i j}\right)\left(\theta_{i j}\right.$ is the misorientation between the pixel $\mathrm{i}$ and its $\mathrm{j}^{\text {th }}$ neighbor). The probability $P_{i j}$ for the growth of pixel $\mathrm{i}$ towards its $\mathrm{j}^{\text {th }}$ neighbor is given by the following equations 3-4:

$$
\begin{array}{ll}
\left.P_{i j}=M\left(\theta_{i j}\right) \llbracket \gamma\left(\theta_{i j}\right)+C \llbracket \Delta H_{i j}\right] & \text { if } \Delta E_{i j}<0 \\
P_{i j}=M\left(\theta_{i j}\right) \llbracket \gamma\left(\theta_{i j}\right)+C \llbracket \Delta H_{i j} \rrbracket \llbracket \exp \left(-\Delta E_{i j} / k T\right) & \text { if } \Delta E_{i j}>0
\end{array}
$$


$C$ is a constant to show the ratio between the influence of the stored energy and that of the GB energy during primary recrystallization. Its value is in the range of 0.1 and 10 [27]. In the present paper, both influences were supposed to have the same level $(\mathrm{C}=1)$.

$\Delta E_{i j}$ is the energy variation after the growth of pixel $\mathrm{i}$ towards its $\mathrm{j}^{\text {th }}$ neighbor (or reorientation of its $\mathrm{j}^{\text {th }}$ neighbor to have the same orientation as i). It can be presented by the variation of GB energy $\Delta E G_{i j}$ and that of the stored energy $\Delta E S_{i j}$ (Equation 5).

$$
\Delta E_{i j}=\Delta E G_{i j}+\Delta E S_{i j}
$$

$\Delta E G_{i j}$ is related to the misorientation variation between the pixels. After the growth, the misorientations between reoriented pixel (as $1^{\text {st }}$ neighbor in figure $4 \mathrm{~b}$ ) and its own neighbors (as A, $\mathrm{B}, \mathrm{C}, \mathrm{i}, 2^{\text {nd }}$ and $6^{\text {th }}$ neighbors in figure $\left.4 \mathrm{~b}\right)$ will be changed. The GB energy $\gamma\left(\theta_{j k}\right)$ will be hence changed (Equation 6). For this reason, during the calculation of $\Delta E G_{i j}$, some second order neighbors of pixel i should be considered (as A, B, C in figure 4b).

$$
\Delta E G_{i j}=\left[\sum_{k=1}^{6} \gamma\left(\theta_{j k}^{\text {final }}\right)-\sum_{k=1}^{6} \gamma\left(\theta_{j k}^{\text {initial }}\right)\right][S
$$

$\gamma\left(\theta_{j k}^{\text {initial }}\right)$ and $\gamma\left(\theta_{j k}^{\text {final }}\right)$ are the GB energies $\left(\mathrm{J}^{-\mathrm{m}^{-2}}\right)$ between reoriented pixel $\left(\mathrm{j}^{\text {th }}\right.$ neighbor $)$ and its own neighbors, respectively before and after the growth of pixel $\mathrm{i}$ towards $\mathrm{j}^{\text {th }}$ neighbor. $\mathrm{S}$ is the surface of the interface between two pixels. It is constant and will be explained later.

$\Delta E S_{i j}$ corresponds to the stored energy variation of the reoriented pixel (Equation 7). For the pixel i which belongs to an existing nucleus, its stored energy is already zero, and the stored energy variation equals to that of the reoriented pixel $\mathrm{j}$.

$$
\Delta E G_{i j}=\left[H_{j}^{\text {final }}-H_{j}^{\text {initial }}\right] V
$$

$H_{j}^{\text {initial }}$ and $H_{j}^{\text {final }}$ are the stored energies $\left(\mathrm{J} . \mathrm{mol}^{-1}\right.$ is modified to be $\left.\mathrm{J} \cdot \mathrm{m}^{-3}\right)$ of reoriented pixel before and after the growth of pixel i. $\mathrm{V}$ is the pixel volume which is constant.

The pixels are supposed to be as three dimensional ones with the acquisition step size considered as the pixel height (Figure 5). Then, the common surface S (red plane) between two pixels and the pixel volume $\mathrm{V}$ can be estimated. 


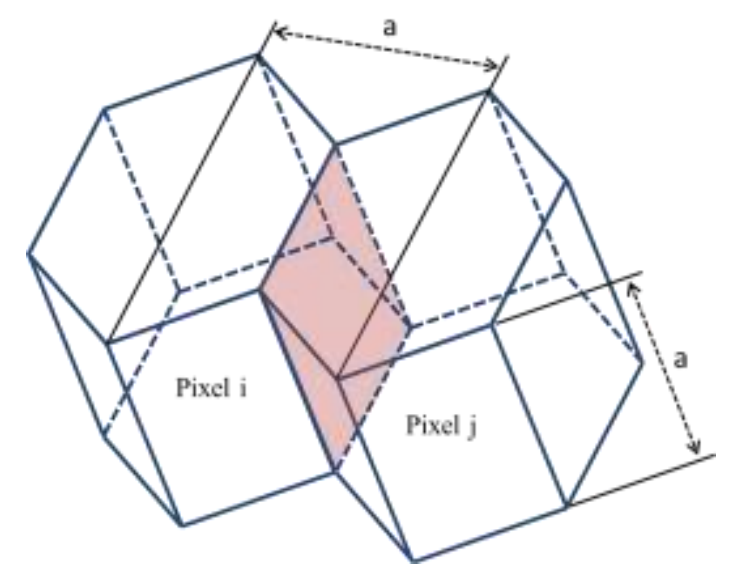

Figure 5: Supposed three dimensional pixels with acquisition step size a considered as the pixel height. The red plane is the common surface between two adjacent pixels.

For the interface mobility $M\left(\theta_{i j}\right)$ between two pixels, a maximum value $\left(M_{\max }=1\right)$ and a minimum value $\left(M_{\min }=0\right)$ are introduced. Two limit values $\gamma_{\max }$ and $\gamma_{\min }$ were also used for the GB energy $\gamma\left(\theta_{i j}\right)$. The GB energy of nickel $0.8 \mathrm{~J} . \mathrm{m}^{-2}$ [28] was chosen as $\gamma_{\max }$, and $\gamma_{\min }=0$.

Both $M\left(\theta_{i j}\right)$ and $\gamma\left(\theta_{i j}\right)$ which are related to the misorientation $\theta_{i j}$, are defined as [16]:

- If $\theta_{i j}<5^{\circ}$, two adjacent pixels were considered as within the same grain, so $M\left(\theta_{i j}\right)=M_{\min }$ and $\gamma\left(\theta_{i j}\right)=\gamma_{\min }$

- If $\theta_{i j}>15^{\circ}$, the interface between two pixels corresponds to a HAGB. The mobility and the energy of HAGB are considered as constant, with $M\left(\theta_{i j}\right)=M_{\max }$ and $\gamma\left(\theta_{i j}\right)=\gamma_{\max }$

- If $5^{\circ} \leq \theta_{i j} \leq 15^{\circ}$,

$$
M\left(\theta_{i j}\right)=1-\exp \left[\left(-\frac{\theta_{i j}}{\theta_{0}}\right)^{3}\right] \text { with } \theta_{0}=15^{\circ}
$$

and $\gamma\left(\theta_{i j}\right)$ is calculated by the Read and Shockley equation [29]:

$$
\gamma\left(\theta_{i j}\right)=\gamma_{\max } \frac{\theta_{i j}}{\theta_{0}}\left[1-\log \left(\frac{\theta_{i j}}{\theta_{0}}\right)\right]
$$


Moreover, the GB energy and mobility for the coherent twin boundaries $\Sigma 3$ coh are almost zero. On the contrary, those of incoherent ones $\Sigma 3$ incoh are not null. However, the determination of the coherence of $\Sigma 3$ GBs (including $\Sigma 3$ coh and $\Sigma 3$ incoh) is feasible but very heavy [30]. Therefore, these two parameters are set to zero for all the $\Sigma 3$ GBs. The $\Sigma 3$ GBs can be easily determined according to the Brandon's definition $\left(60^{\circ}\right.$ about a common $<111>$ crystal axis with $8.7^{\circ}$ as the maximum deviation [31]).

After the calculation of the growth probability $P_{i j}$, the maximum probability will be chosen as the growth direction of the pixel i. If several neighbors have simultaneously the maximum probability, the pixel i will grow along one direction chosen randomly among them.

\subsection{Twinning}

\subsubsection{Twinning with formation of $\Sigma 3 \mathrm{coh}$ boundary}

From Gleiter's theory [3] and the TEM observation [13], the active twinning system seems to depend on the direction of grain growth. Therefore, in this simulation, the growth direction of a growing grain will be firstly detected according to the process described in section 3.1 (blue arrow towards $6^{\text {th }}$ neighbors in figure $\left.6 a\right)$. Then, the directions of its $4<111>$ axes will be also analyzed (green lines in figure 6a). If there is at least one $<111>$ axis that approaches the growth direction (dotted green line), it is possible to have twinning from this growing grain. If more than one $<111>$ axis is aligned with this direction, the closest one will be taken as the twinning system.

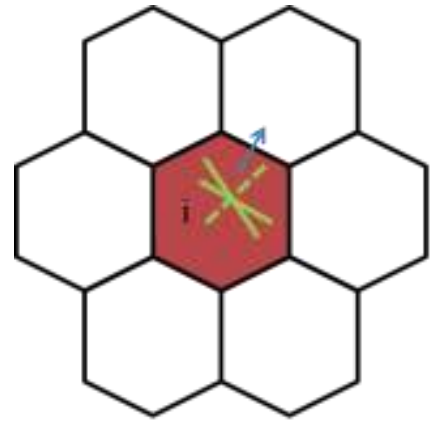

(a)

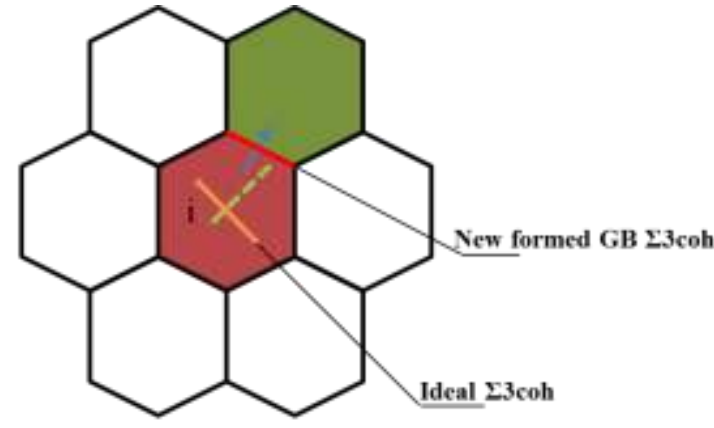

(b)

Figure 6: Twinning model: (a) comparison of <111> crystal axes (green lines) with chosen growth direction (blue arrow) and (b) twinning with the axis the most close to growth direction (dotted green line). The orange line indicates the theoretical $\Sigma 3$ coh orientation which is perpendicular to the twinning axis. The red line shows the simulated $\Sigma 3$ coh after twinning. 
In addition, during the annealing twin formation, a new $\Sigma 3$ coh boundary will be formed. The GB $\Sigma 3$ incoh are formed as the indirect outcomes after the formation of coherent ones $\Sigma 3 \operatorname{coh}[13]$.

There are two conditions for a boundary to be considered as a $\Sigma 3$ coh boundary between two grains:

- The misorientation of this interface should correspond to a $60^{\circ}$ rotation around a common $<111>$ axis (as the definition of $\Sigma 3$ ) and

- The plane of the $\Sigma 3$ coh boundary should be parallel to a common $\{111\}$ plane of both grains, or perpendicular to the common $<111>$ axis of rotation.

To state on the coherence of $\Sigma 3$ boundary, both previous conditions must be checked. The first condition can be verified by the twin orientation calculation (as in figure $6 \mathrm{~b}, 6^{\text {th }}$ neighbors is reoriented with the twin orientation of pixel i) [32]. For the second condition, the orientation of the boundary plane should be considered and compared to a $\{111\}$ plane. Nevertheless, this is not easy to calculate the boundary plane (or plane trace) from an EBSD map. As shown in figure 6b, the new formed $\Sigma 3$ coh boundary (red line) is not perpendicular to the common $<111>$ axis, and it deviates thus from the ideal $\Sigma 3$ coh direction (orange line). This deviation is induced by the hexagonal pixel shape.

For the EBSD map which contains hexagonal pixels, the grain boundaries can be defined by OIM in two ways: either a serie of segments between the adjacent pixels (red line in figure 7) or a straight line between two triple points (blue line in figure 7).

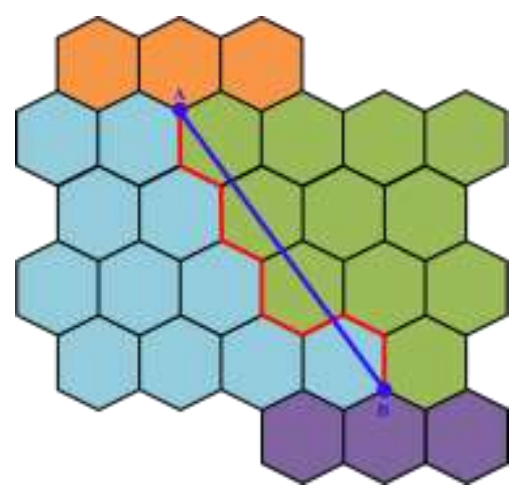

Figure 7: GB definitions by OIM. The red line shows the definition with a series of pixel segments. The blue line presents the GB as a straight line between two triple points.

In this simulation, the new formed GB is as short as one segment between two pixels (Figure 
8a). Both GB definitions of OIM are the same, and this short GB has only three possible directions (as a, b and $\mathrm{c}$ shown in figure $8 \mathrm{a}$ ). But, for the real situation, the ideal $\Sigma 3 \mathrm{coh}$ boundary could be oriented towards any direction. Consequently, it is almost impossible to simulate the ideal $\Sigma 3 \mathrm{coh}$ boundary with limited GB directions at the first stage of twin formation.

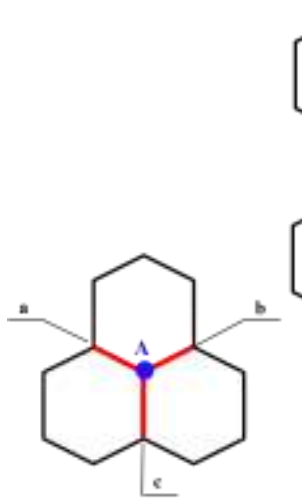

(a)

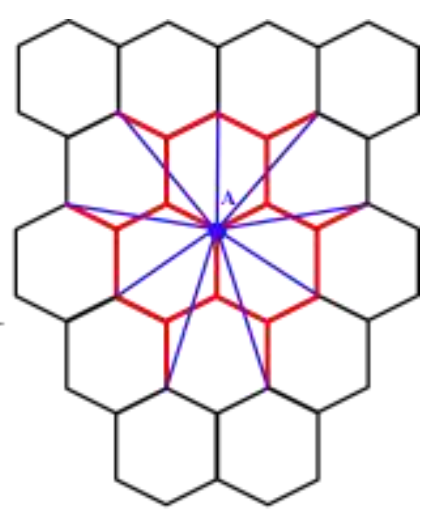

(b)

Figure 8: Short grain boundaries (one segment) and long grain boundaries (three segments) from a triple point A. the red lines and blue lines show respectively two GB definitions.

For example, if the GB corresponds to three segments (Figure $8 b$ ), both definitions become different. The number of possible GB directions increases. By analogy, the more the GB is long, the more possible directions have the GBs [30]. Then, it becomes easier to have the GBs parallel to the $<111>$ axes.

Therefore, in our simulation, the twinning will be firstly realized according to the $<111>$ axis of parent grain that is close to its growth direction, with the formation of a $\Sigma 3$ coh boundary (black line in figure 9b). During the growth of the parent grain and its twin, the length of the formed $\Sigma 3 \mathrm{coh}$ will increase. At the same time, the orientation of the $\Sigma 3 \mathrm{coh}$ will be adjusted by comparison to the ideal one (Figure 9c-d). 


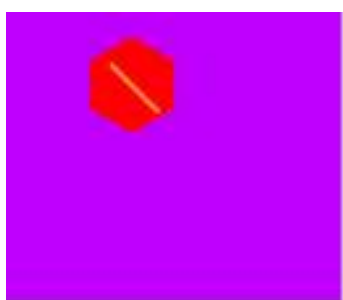

(a)

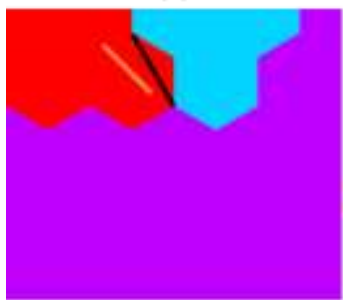

(c)

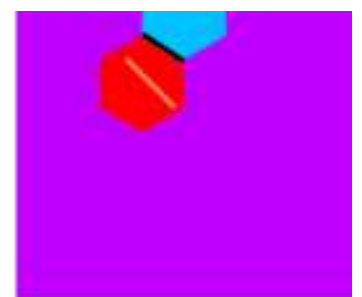

(b)

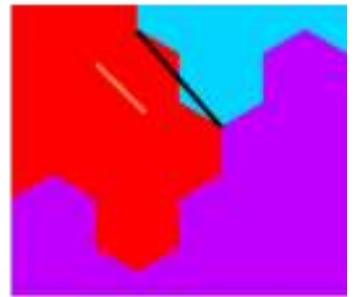

(d)

Figure 9: An example for (a) a recrystallized grain (red pixel) surrounded by the deformed area (purple); (b) twinning with formation of a short $\Sigma 3 \mathrm{coh}$ boundary; (c-d) growth of parent grain and its twin (blue pixel) with adjustment of $\Sigma 3$ coh boundary compared with the ideal one (orange line) In this figure, the $\Sigma 3 \mathrm{coh}$ boundary is defined by the second method presented in figure 7 .

\subsubsection{Multiple twinning}

In fact, during the experimental annealing, the twin formation corresponds to multiple twinning. It is revealed by the presence of several $\Sigma 3 \mathrm{coh}$ boundaries or twin parts in the same recrystallized grain. These twin boundaries are either parallel to (retwinning [32]) or intersecting with each other.

The stored energy effect on twin formation has been already observed in the same alloy [19]. It was found that the twinning depends on the stored energy $E$ (or strain amount) and the grain size $D$ (Equation 10)

$$
N=(A \llbracket E+B)\left(D-D_{0}\right)
$$

$A$ and $B$ are constants. $N$ is the $\Sigma 3$ boundary (including $\Sigma 3$ coh and $\Sigma 3$ incoh) number per grain. $D_{0}$ is the critical grain size for the twin formation.

For multiple twinning, a simplified equation 10 was employed (Equation 11).

$$
N=A^{\square}\left(D-D_{0}\right)
$$

Indeed, since the simulation is realized for only one deformation rate, $(\mathrm{AE}+\mathrm{B})$ in replaced by 
the constant $\mathrm{A}^{\prime}$, and $\mathrm{N}$ is considered as ideal $\Sigma 3$ coh boundary number or twinning times of a grain. Then the twin formation was only controlled by the grain size. For a grain of size $\mathrm{D}$, if the existing $\Sigma 3$ coh boundary number is smaller than the experimental value $\mathrm{N}$, the new twinning is authorized during its growth.

\section{Results and discussion}

\subsection{Recrystallization}

This simulation is used to simulate the recrystallization and twinning of a deformed alloy during an annealing at $900^{\circ} \mathrm{C}$. Experimentally, the recrystallization is very fast, and the recovery is negligible [19].

\subsubsection{Nucleation}

The figure 10 presents the nucleation after one step of MC simulation. It shows the nuclei are formed close to the area with strong local deformation (black areas). The distribution of these nuclei is homogenous within the deformed microstructure.

This homogenous distribution of nucleus is due to the lack of recovery process during simulation which controls the location and moment of nucleation.

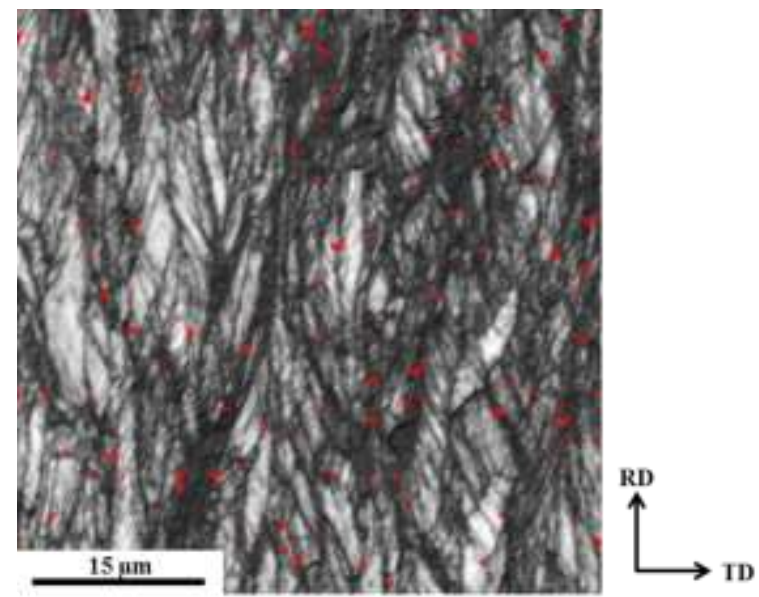

Figure 10: IQ map for the distribution of nuclei after one step of $M C$ simulation (nuclei are shown by red points). 


\subsubsection{Kinetic of recrystallization}

The recrystallized fraction during simulation is plotted in figure 11, as a function of the MC step (on log scale). From the Johnson-Mehl-Avrami-Kolmogorov (JMAK) equation, the Avrami exponent $\mathrm{n}$ is 2 . This simulated value is close to the theoretical one which should be in the range between 2 and 3 for a two dimensional growth [33]. This theoretical value range is obtained on the basis of a homogenous distribution of nuclei during recrystallization. The experimental value is always smaller than 2 [33]. This is due to the heterogeneous distribution of nuclei, as effect of shear band which is a favor site for nucleation. For our simulation, the nuclei distribution is homogeneous (Figure 10). During experimental annealing, the distribution of nuclei is heterogeneous with formation of nucleus clusters. Their growth will be blocked by each other during the recrystallization because of the null stored energy gradient. This growth restraint delays the recrystallization kinetic. Therefore, the bigger value of $\mathrm{n}$ compared with experimental one is reasonable.

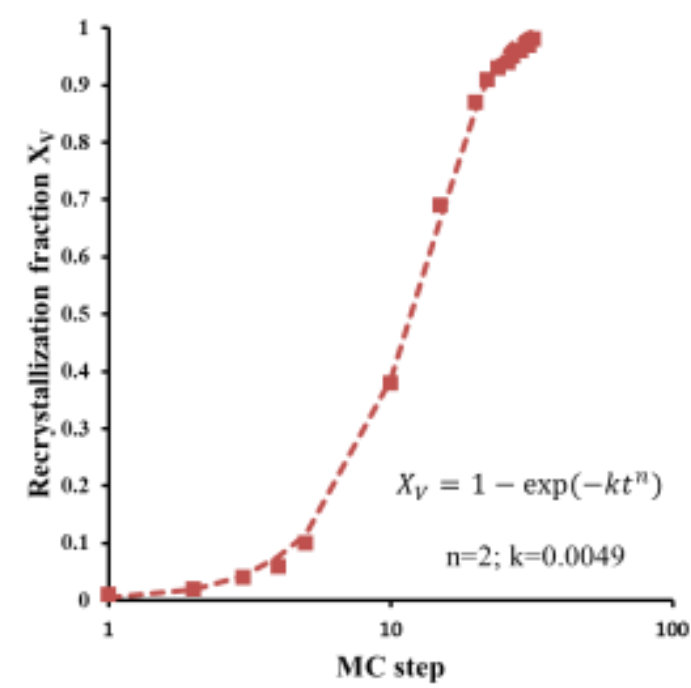

Figure 11: Kinetic of recrystallization obtained by MC simulation.

Moreover, from JMAK equation, the pre-exponential factor $\mathrm{k}$ is 0.0049 which depends on the temperature [34]. However, it is difficult to compare this simulated parameter with experimental one. Because the recrystallization during simulation is in function of MC steps, but that during experiment is related to time. 


\subsubsection{Microstructure after complete recrystallization}

The recrystallized microstructures after simulation and an annealing of 4 min at $900^{\circ} \mathrm{C}$ (experiment) are shown in figure 12. Within these microstructures, the colors are used only to separate the grain without considering the twin boundaries. One can find that the simulated microstructure is similar to the experimental one. However, the grains within experimental microstructure seem to be bigger than simulated ones. Therefore, more statistical analyses as the mean grain size and the grain size distribution were carried out for the experimental microstructure. These analyses are done from an EBSD map of $150 \times 350 \mu \mathrm{m}^{2}$ for a statistical result. Nevertheless, the same analyses were also done for the simulation, from the total simulated area of $50 \times 50 \mu \mathrm{m}^{2}$. The mean grain sizes are 4.7 and $4.1 \mu \mathrm{m}$ for simulation and experiment, respectively. Then, the mean grain size is almost identical for both microstructures.
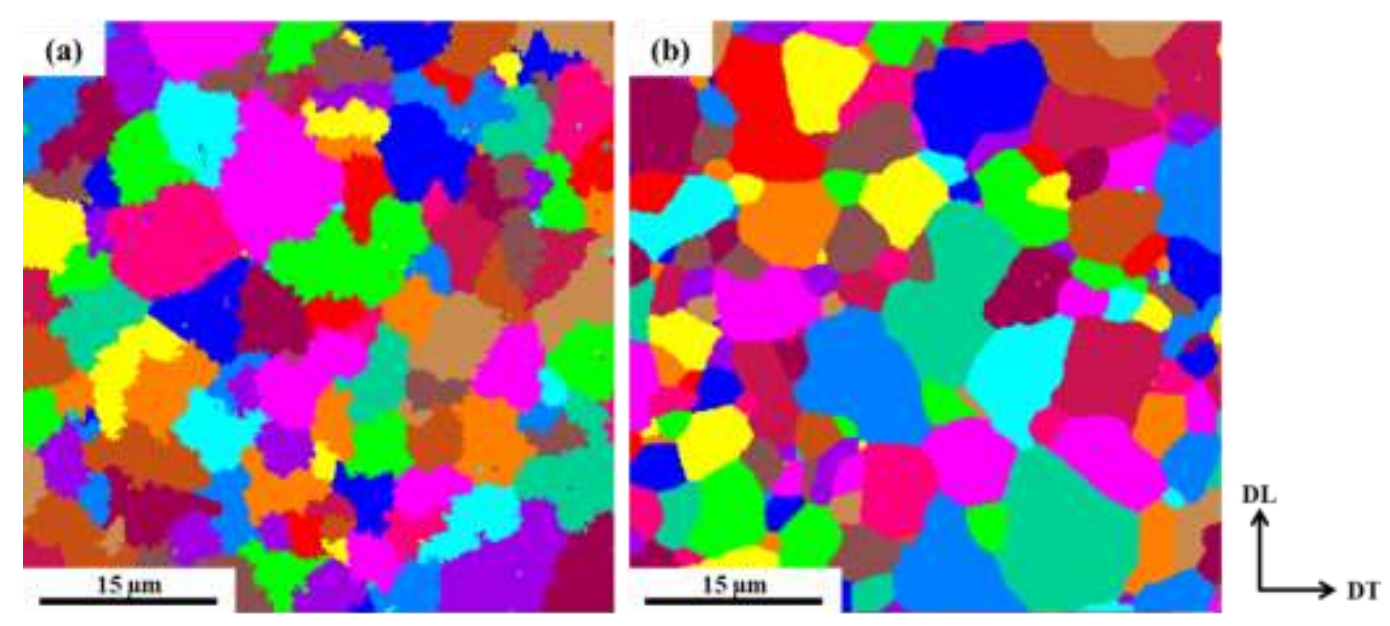

Figure 12: Microstructure after (a) simulation and (b) annealing of 4 min at $900^{\circ} \mathrm{C}$. The colors are used only to distinguish the grains without considering the twin boundaries.

The grain size distributions of both microstructures are shown in figure 13. From the distribution curves, there is a difference between the peak values of both distributions. The experimental microstructure presents more small grains and more coarse grains (black arrows) than simulated one. One can assume that after complete recrystallization, some grains grow by consuming some others that become small. This grain growth is not introduced in the simulation, and then the recrystallized grains cannot thus grow at the expense of others. 


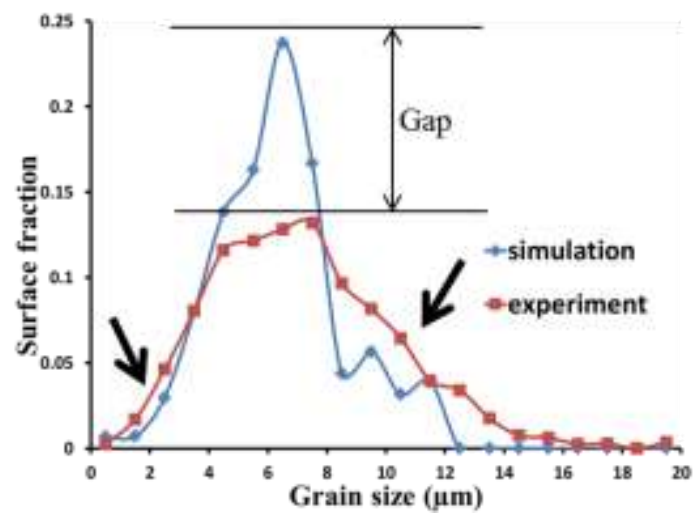

Figure 13: Grain size distribution after simulation and annealing of 4 min at $900^{\circ} \mathrm{C}$ (experiment).

\subsection{Twinning}

\subsubsection{Morphology of twin boundaries}

Four morphologies of annealing twins and thus the twin boundaries (Figure 14a) have been presented by Mahajan et al. [5]. A is a twinned area at the corner of the parent grain with one $\Sigma 3$ coh boundary (red line). B is a twin traversing the grain with two parallel $\Sigma 3$ coh boundaries. C is a twin which terminates in the grain. It is surrounded by two $\Sigma 3 \mathrm{coh}$ boundaries and a $\Sigma 3$ incoh (green line). As the twin formation is three dimensional, the situation $\mathrm{D}$ which locates inside of the parent grain is also possible. It corresponds to the trace of a twin.

Besides these situations above, two other morphologies were also observed by MET studies of the same alloy (Figure 14b and c) [13]. E is a twinned part that traverses the grain. However, the interface between it and the parent grain correspond to two $\Sigma 3$ coh linked by a $\Sigma 3$ incoh. It was also found that the parent grain can twin towards different directions with formation of two twinned parts as F and G. When they meet together during their growth, a $\Sigma 9$ (blue line) is formed. For this situation, the $\Sigma 3 \mathrm{coh}$ boundaries are intersecting and so are not parallel. 


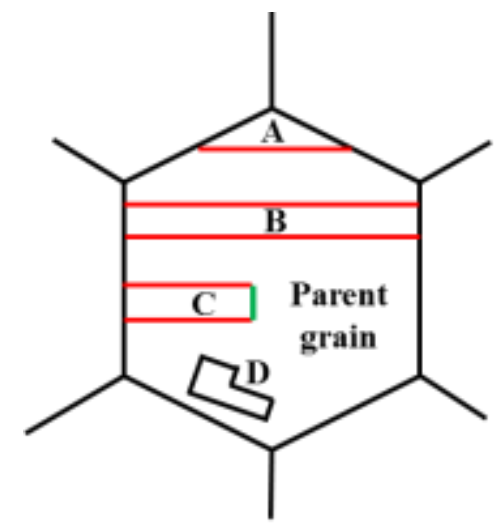

(a)

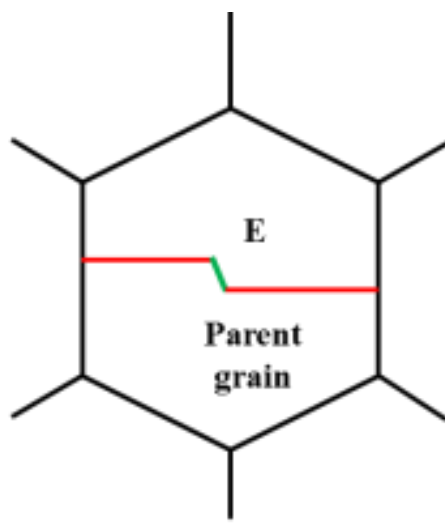

(b)

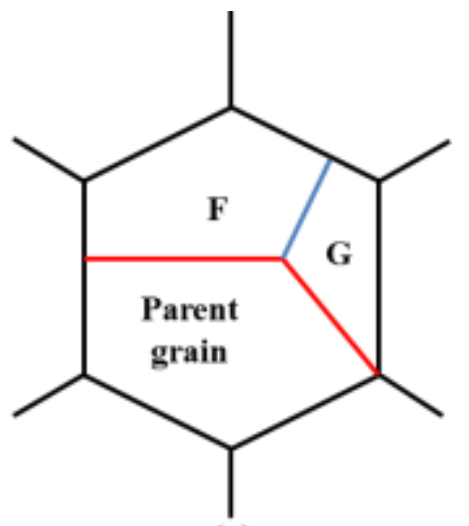

(c)

Figure 14: (a) Four different morphologies of twin parts presented by Mahajan et al. [5], (b) and (c) two other morphologies found during MET study of the same alloy. The red, green and blue lines show respectively, the $\Sigma 3 \mathrm{coh}, \Sigma 3$ incoh and $\Sigma 9$ boundaries.

The simulated distribution of GBs after complete recrystallization (35 MCs) is shown in figure 15. The $\Sigma 3$ coh boundaries (green lines) can be observed in the grains. They are always straight.

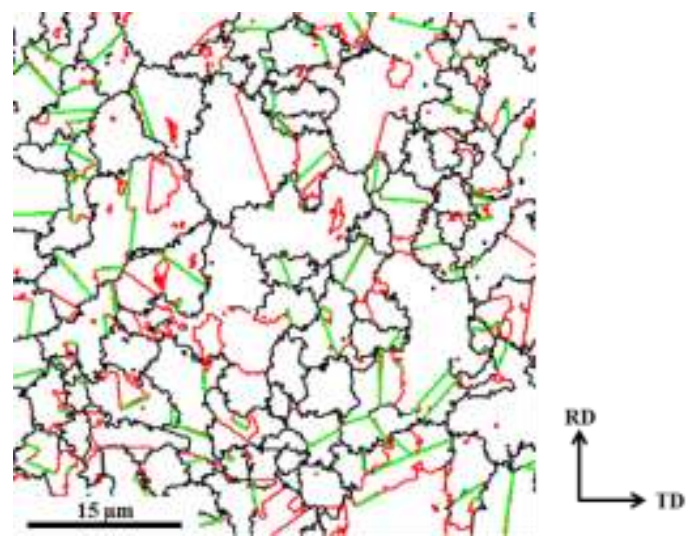

Figure 15: Distribution of GBs within the recrystallized microstructure. The black, red and green lines show the HAGBs, $\Sigma 3$ and $\Sigma 3$ coh boundaries (detected by $\mathrm{OIM}^{\mathrm{TM}}$ ).

The figure 16 shows the detailed morphologies obtained by simulation. The red lines show all the $\Sigma 3$ boundaries including $\Sigma 3 \mathrm{coh}$ and $\Sigma 3$ incoh ones. The green lines show only the $\Sigma 3 \mathrm{coh}$ boundaries.

From this figure, all the experimental morphologies shown in figure 14 are reproduced by simulation. The figure 16a shows the situation B. The figure $16 \mathrm{~b}$ presents the morphology F-G with two $\Sigma 3 \mathrm{coh}$ and one $\Sigma 9$ from a triple point (blue arrow). The figure $16 \mathrm{c}$ shows the simulations of twins A and C (orange arrow reveal the $\Sigma 3$ incoh boundary). Finally, the situations E and D are 
also obtained (Figure 16d). As only the $\Sigma 3$ coh boundary formation is introduced in this simulation, the presence of the $\Sigma$ incoh and $\Sigma 9$ boundaries is an indirect outcome. Indeed, the $\Sigma 3$ incoh and $\Sigma 9$ boundary formation corresponds to the association of two $\Sigma 3$ coh boundaries. This phenomenon is largely studied during grain boundary engineering (GBE) [35].

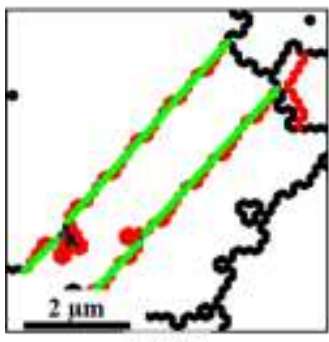

(a)

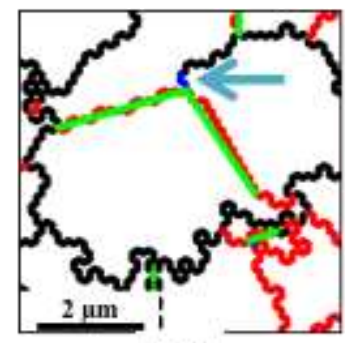

(b)

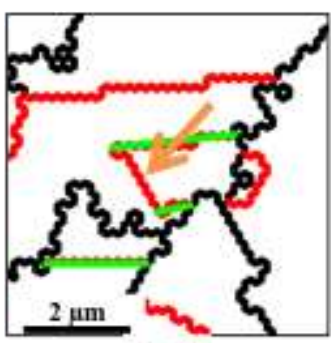

(c)

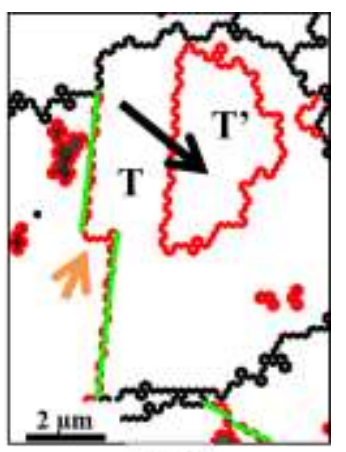

(d)

Figure 16: Detailed morphologies of twinned parts obtained by simulation. The black, red and green lines show the same GBs as figure 15. The blue and orange arrows show $\Sigma 9$ and $\Sigma$ 3incoh, respectively.

From this simulation, the formation of situation E (Figure 14b) can be explained by figure 17a. During the growth of parent grain 1, one side (on the right) forms a twinned part $\mathrm{E}$ and creates a $\Sigma 3$ coh boundary (green line). On the contrary, the other side (on the left) of parent grain 1 forms a twin later and at another location. When the two twinned parts E meet together, the $\Sigma$ 3incoh boundary is formed. Therefore, the situation E can be considered as the result of two $\Sigma 3$ coh formed at different moment or different location.

The situation $\mathrm{C}$ with presence of a $\Sigma 3$ incoh boundary is similar to the situation $\mathrm{E}$. The schema of this case is shown in figure 17b. During the growth of parent grain 1, the twinned part $\mathrm{C}$ is formed from the right side as situation $\mathrm{E}$ with creating a $\Sigma 3$ coh boundary. On the contrary, instead of twin formation, the other side (on the left) of parent grain 1 continues to grow. At the same time, the twinned part $\mathrm{C}$ grows also. During its growth, the area $\mathrm{C}$ twins and develops another parallel $\Sigma 3 \mathrm{coh}$ boundary. The $\Sigma 3$ incoh boundary is also formed automatically as a geometrical product. This retwinning process allows the formation of an area (parent grain 2) with the same orientation as parent grain 1. During the growth of both parent grain parts, they meet 
each other and a closed "circle" grain is formed. Therefore, the situation C is the result of twice twinning at one side of parent grain and the growth of parent grain surrounding the twinned part C.

From situations $\mathrm{E}$ and $\mathrm{C}$, one can find, the $\Sigma 3$ incoh boundary was well simulated as an indirect product which is related to formation of $\Sigma 3$ coh ones.
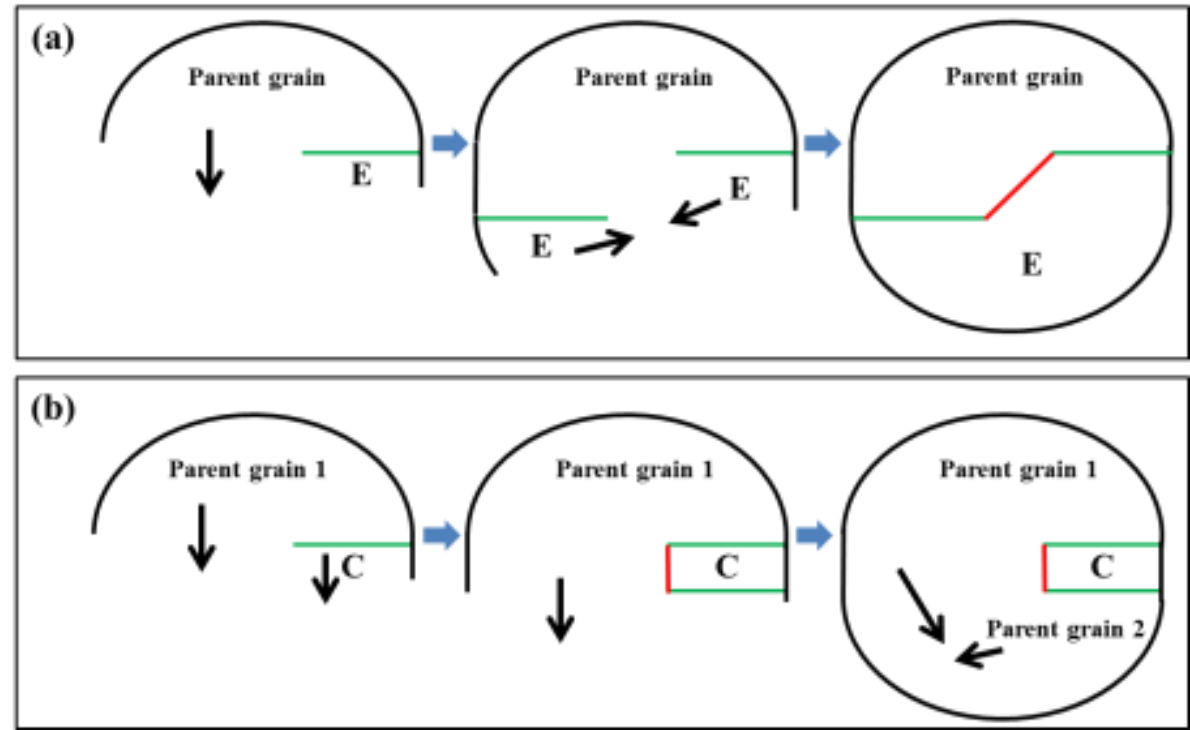

Figure 17: Schemas for the formation of situations (a) E and (b) C, shown in figure 14.

The situation $\mathrm{D}$ in figure $14 \mathrm{a}$ is considered as the trace of a twin part, because the twin formation is three dimensional. However, our simulation is two dimensional, so the formation of this situation (twin part T' indicated by black arrow) as shown in figure $16 \mathrm{~d}$ is not physical. In fact, the area $\mathrm{T}^{\prime}$ is formed fist by twinning from $\mathrm{T}$ (straight boundary). Then the growth of $\mathrm{T}$ surrounds it with formation of a closed area T'. This simulated twin T' is surrounded by a straight $\Sigma 3 \mathrm{coh}$ boundary and a semi-circle $\Sigma 3$ incoh boundary. Let us note, that as these two boundaries form a closed "circle", they cannot be separated by the OIM analysis and the coherence of the $\Sigma 3$ cannot thus be detected (Figure 16d).

\subsubsection{Twinning frequency}

The twinning frequency is studied by analysis of $\Sigma 3$ (including $\Sigma 3$ coh and $\Sigma 3$ incoh) fraction and its density. The definition of these two parameters has been already presented in [19]. The 
corresponding values obtained from simulation and experiment after 4 min annealing at $900^{\circ} \mathrm{C}$ are shown in table 1.

Table 1: $\Sigma 3$ fraction and its density obtained by simulation and experiment

\begin{tabular}{lcc}
\hline & $\boldsymbol{\Sigma}$ fraction & $\boldsymbol{\Sigma}$ 3 density $\left(\boldsymbol{\mu m}^{-\mathbf{1}}\right)$ \\
\hline Simulation & $30.7 \%$ & 0.37 \\
\hline Experiment & $51.5 \%$ & 0.54 \\
\hline
\end{tabular}

Both parameters reveal the twinning frequency is lower during simulation than experiment. This is because the twinning during simulation is controlled by two conditions:

- the evolution of twin formation (Equation 11) which is obtained through experiments;

- the presence of at least one <111> crystal axis of parent grain close to its growth direction, itself chosen by probability calculation.

For the experimental situation, as shown in figure 18a, the grain 1 can grow towards grain 2 simultaneously in all directions with the same probability (red arrows). Then it is easy to have one $<111>$ axis (green lines in pixel i) that is parallel to the growth direction for twin formation. On the contrary, during the MC simulation, only one growth direction is chosen. It is thus possible to have none of corresponding <111> axis (Figure 18b). Therefore, the used model decreases the twin frequency during simulation.

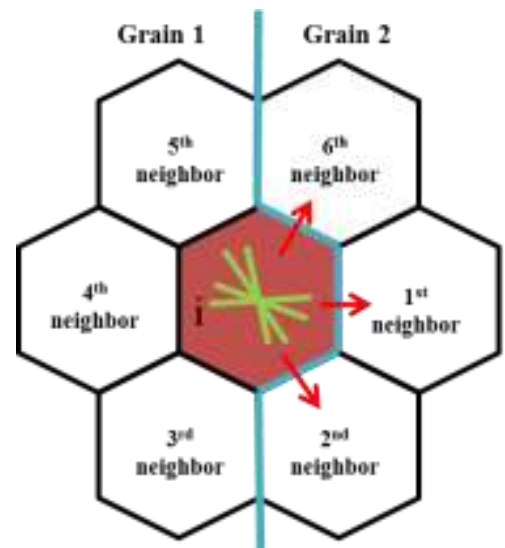

(a)

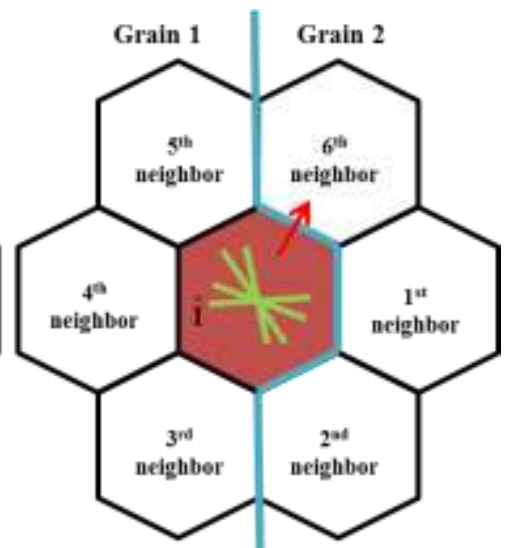

(b)

Figure 18: (a) Real growth simultaneously in several directions with the same probability and (b) MC simulation with only one chosen direction. The blue lines show the HAGBs. The green lines 
reveal the $4<111>$ axes of growing pixel i. The red arrows show the possible growth directions.

\section{Conclusions}

The MC simulation of primary recrystallization at $900^{\circ} \mathrm{C}$ was realized for a deformed nickel alloy. Without recovery process, the simulated nucleation is homogeneous. This favors an Avrami exponent $\mathrm{n}$ close to the ideal value, but deviated from the experimental one. Moreover, the recrystallization kinetic is in agreement with experimental results. After complete recrystallization, because the grain growth is not introduced in the simulation, a difference of grain size distribution between simulation and experiment is observed. However, the mean grain size are similar.

In addition, the annealing twinning was also simulated at a microscopic scale. Different morphologies observed during experiments were simulated. Therefore, the annealing twin process can be considered as a mechanism of primary recrystallization. The dependence of grain growth direction on twin formation reveals the possibility of parallel $\Sigma 3$ coh boundaries formation. Moreover, it showed the $\Sigma 3$ incoh and $\Sigma 9$ boundaries are the indirect outcome after $\Sigma 3$ coh formation. A smaller twin frequency is obtained during simulation compared with experiment because at this time, only one grain growth direction is taken into account in the simulation.

\section{Acknowledge}

The authors would like to thank Denis SOLAS, Isabelle DROUELLE and Thierry AUGER for fruitful discussions, and thank Aubert \& Duval for providing materials. 


\section{References}

[1] J.A. Ewing, W. Rosenhain, Philos T Roy Soc A. 195 (1900) 279.

[2] R.L. Fullman, J.C. Fisher, J Appl Phys. 22 (1951) 1350.

[3] H. Gleiter, Acta Metall. 17 (1969) 1421.

[4] M.A. Meyers, L.E. Murr, Acta Metall. 26 (1978) 951.

[5] S. Mahajan, C.S. Pande, M.A. Imam, B.B. Rath, Acta Mater. 45 (1997) 2633.

[6] B.B. Rath, M.A. Imam, C.S. Pande, Mater. Phys. Mech. 1 (2000) 61.

[7] H. Hofmeister, In: H.S. Nalwa (Eds.), Encyclopedia of Nanoscience and Nanotechnology, Volume 3, American Scientific Publishers, California, 2004, pp.431.

[8] D. Farkas, E. Bringa, A. Caro, Phys Rev B. 75 (2007) 184111.

[9] E.M. Bringa, D. Farkas, A. Caro, Y.M. Wang, J. McNaney, R. Smith, Scripta Mater. 59 (2008) 1267.

[10] C.S. Pande, M.A. Imam, B.B. Rath, Metall Trans A. 21 (1990) 2891.

[11] J.R. Cahoon, Q. Li, N.L. Richards, Mat Sci Eng A. 526 (2009) 56.

[12] Q. Li, J.R. Cahoon, N.L. Richards, Mat Sci Eng A. 527 (2009) 263.

[13] W. Wang, Etude des mécanismes de recristallisation de nouveaux alliages à base de Ni, Ph.D. at Université Paris Sud, Orsay, 2014.

[14] F. Caleyo, T. Baudin, R. Penelle, Scripta Mater. 46 (2002) 829.

[15] T. Baudin, F. Julliard, P. Paillard, R. Penelle, Scripta Mater. 43 (2000) 63.

[16] P. Volovitch, F. Caleyo, T. Baudin, P. Gerber, R. Penelle, C. Rey, Mater Sci Forum. 495-497 (2005) 507.

[17] S.H. Choi, J.H. Cho, Mat Sci Eng A. 405 (2005) 86.

[18] Y.B. Chun, S.L. Semiatin, S.K. Hwang, Acta Mater. 54 (2006) 3673.

[19] W. Wang, F. Brisset, A.L. Helbert, D. Solas, I. Drouelle, M.H. Mathon, T. Baudin, Mat Sci Eng A. 589 (2014) 112.

[20] A.L. Etter, M.H. Mathon, T. Baudin, V. Branger, R. Penelle, Scripta Mater. 46 (2002) 311-317.

[21] A.L. Etter, T. Baudin, M.H. Mathon, W. Swiatnicki, R. Penelle, Scripta Mater. 54 (2006) 683-688.

[22] L.N. Brewer, D.P. Field, C.C. Merriman, In: A.J. Schwartz, M. Kumar, B.L. Adams, D.P. Field (Eds.), Electron Backscatter Diffraction in Materials Science, Second Ed., Springer, New York, 2009, pp.251.

[23] M. Calcagnotto, D. Ponge, E. Demir, D, Raabe, Mat Sci Eng. A. 527 (2010) 2738.

[24] K. Pawlik, J. Pospiech, K. Lücke, Texture Microstruct. 14-18 (1991) 25.

[25] D.P. Landau, K. Binder, A guide to Monte-Carlo simulations in statistical physics, third Ed., CAMBRIDGE, New York, 2009.

[26] A.D. Rollett, P. Manohar, In: D. Raabe, F. Roters, F. Barlat, L.Q. Chen (Eds.), Continuum scale simulation of engineering materials: Fundamentals-Microstructures-Process application, WILEY-VCH, Weinheim, 2004, pp.77.

[27] P. Volovitch, T. Baudin, R. Penelle, F. Caleyo, L. Barrallier, Mater Sci Forum. 550 (2007) 453. 
[28] L.E. Murr, Interfacial phenomena in metals and alloys, Addison-Wesley, Reading, MA, 1975. [29] W.T. Read, Dislocations in crystals, McGraw Hill, New York, 1953.

[30] S.I. Wright, R.J. Larsen, J Microsc. 205 (2002) 245.

[31] D.G. Brandon, Acta Metall. 14 (1966) 1479.

[32] G. Gottstein, Acta Metall. 32 (1984) 1117.

[33] F.J. Humphreys, M. Hatherly, Recrystallization and related annealing phenomena, Second Ed., Elsevier, Oxford, 2004.

[34] M. Avrami, J Chem Phys. 7 (1939) 1103.

[35] V. Randle, Acta Mater. 52 (2004) 4067. 